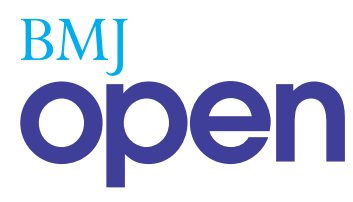

\title{
Predicting and preventing postconcussive problems in paediatrics (5P) study: protocol for a prospective multicentre clinical prediction rule derivation study in children with concussion
}

\author{
Roger Zemek, ${ }^{1,2}$ Martin H Osmond, ${ }^{1,2}$ Nick Barrowman, ${ }^{2}$ on behalf of the the \\ Pediatric Emergency Research Canada (PERC) Concussion Team
}

To cite: Zemek R, Osmond MH, Barrowman N, et al. Predicting and preventing postconcussive problems in paediatrics $(5 \mathrm{P})$ study: protocol for a prospective multicentre clinical prediction rule derivation study in children with concussion. BMJ Open 2013;3:e003550. doi:10.1136/bmjopen-2013003550

- Prepublication history and additional material for this paper is available online. To view these files please visit the journal online (http://dx.doi.org/10.1136/ bmjopen-2013-003550)

Received 4 July 2013 Accepted 5 July 2013

\section{${ }^{1}$ Departments of Pediatrics and Emergency Medicine, University of Ottawa, Ottawa, Ontario, Canada ${ }^{2}$ Children's Hospital of Eastern Ontario Research Institute, Ottawa, Ontario, Canada}

\footnotetext{
Correspondence to Dr Roger Zemek; rzemek@cheo.on.ca
}

\begin{abstract}
Introduction: Persistent postconcussive symptoms (PCSs) is the persistence of somatic, cognitive, physical, psychological and/or behavioural changes lasting more than 1 month following concussion. Persistent concussion impacts the quality of life through impaired cognition, memory and attention affecting school performance, mood and social engagement. No large epidemiological studies have determined the true prevalence of persistent concussion symptoms. Validated, easy-to-use prognosticators do not exist for clinicians to identify children at highest risk. The goal of Predicting and Preventing Postconcussive Problems in Pediatrics study is to derive a clinical prediction rule for the development of persistent postconcussion symptoms in children and adolescents presenting to emergency department following acute head injury.
\end{abstract}

Methods and analysis: This study is a prospective, multicentre cohort study across nine academic Canadian paediatric emergency departments. We will recruit the largest prospective epidemiological cohort of children with concussion. Eligible children will be followed using Post-Concussion Symptom Inventory, a validated tool in children as young as 5 years. Patients will follow-up at 1, 2, 4, 8 and 12 weeks postinjury. The main outcome will be the presence/absence of PCSs defined as three or more persistent concussion symptoms 1 month following the injury. 1792 patients provide adequate power to derive a clinical decision rule using multivariate analyses to find predictor variables sensitive for detecting cases of persistent postconcussion symptoms.

Ethics and dissemination: Results of this large prospective study will enable clinicians to identify children at highest risk, optimise treatment and provide families with realistic and appropriate anticipatory guidance. Ethics has been obtained through the Children's Hospital of Eastern Ontario Research Ethics Board. Results will be disseminated at international conferences and in four manuscripts to peer-reviewed journals.

\section{ARTICLE SUMMARY}

Article focus

- This article describes the protocol for the development of a clinical prediction rule to determine those children and adolescents at high risk for developing persistent postconcussion symptoms (PCS).

Key messages

- Validated, easy-to-use prognosticators do not exist for clinicians to identify children at highest risk for PCS.

- Physicians currently can accurately neither inform children and parents whether they should expect longer symptoms nor initiate pharmacotherapy or other management to reduce the occurrence or severity of PCS.

Strengths and limitations of the study

- This work will be the largest epidemiological study on paediatric concussion and will provide rigorous evidence to determine PCS incidence in children and its impact on quality of life.

- The results of this large multicentre study will enable clinicians to identify children at highest risk for PCS, optimise treatment and provide families with realistic anticipatory guidance.

- Not all children with acute concussions report to paediatric emergency department which may affect applicability to other settings.

Trial registration: This study is registered at Clinicaltrials.gov through the US National Institute of Health/National Library of Medicine (NCT01873287; http://clinicaltrials.gov/ct2/show/NCT01873287).

\section{INTRODUCTION}

After years of minimising the impact of concussion, there is now a recognition that there is nothing termed mild about mild traumatic 
brain injury. ${ }^{1}{ }^{2}$ Concussion, a 'mild' traumatic brain injury common in children and adolescents, is a complex pathophysiological process affecting the brain induced by traumatic biomechanical forces. ${ }^{3}$ Recent estimates reveal that 1 in 220 paediatric emergency department (ED) visits is for concussion yielding 700000 paediatric concussion ED visits annually in the USA. ${ }^{4-6}$ Census data across eight paediatric EDs across Canada suggest that a higher incidence of 1 in 70 paediatric ED visits is for concussion. Center for Disease Control statistics demonstrate that the majority of concussions occur in children and young adults. ${ }^{7}$

While many children improve within 2 weeks postconcussion, symptoms may persist for months and even years, with children and adolescents at highest risk. ${ }^{3}$ 8-11 When children and adolescents suffer persistent somatic, cognitive, psychological and/or behavioural changes for 1 month duration or longer following a concussion, it is referred to as persistent postconcussive symptoms (PCS). ${ }^{12}$ No large epidemiological studies have determined the true prevalence, but the literature suggests that up to $59 \%$ of children are still symptomatic 1 month following concussion. ${ }^{13-15}$ PCS impacts the quality of life for both the patient and the entire family resulting in school absenteeism, emotional changes and loss of peer activities. ${ }^{9} 1617$ Children with PCS may miss weeks or even months out of the school-year, affecting marks and jeopardising promotion to the next grade. ${ }^{2}{ }^{3}$ In PCS, cognition may be slowed, attention and memory impaired, making schoolwork a challenge on return to school, resulting in de novo educational interventions. ${ }^{9} 1819$

The lay press has become inundated with high-profile athletes suffering tragic consequences following concussion, highlighting an increased risk of dementia, chronic traumatic encephalopathy and suicide. ${ }^{1}$ Children are not immune to life-altering consequences following concussion. ${ }^{20-22}$ Repeated concussions suffered during school-aged years have the potential for permanent cognitive dysfunction and neuropsychiatric problems and there is evidence indicating that sustaining one concussion leads to an increased risk of sustaining others. ${ }^{23-29}$ We need rigorous evidence, beyond anecdotal speculation, about the impact of PCS on the quality of life for children and families. Clinical prediction rules are necessary to determine who will most benefit from future interventional studies designed to reduce the frequency and severity of PCS versus the current standard treatment of rest. ${ }^{3} 3031$

A recent systematic review confirmed that validated, easy-to-use prognosticators do not exist for clinicians to identify children with concussion who are at the highest risk for PCS and sequelae. ${ }^{30}$ Physicians do not have the ability to accurately inform children and parents about symptom duration, hindering the capacity to initiate pharmacotherapy and new management approaches in order to prevent the occurrence or reduce the severity or duration of PCS. ${ }^{1}{ }^{17} 30$ The goal of this study is to derive a clinical prediction rule from key factors that is predictive of PCS development. We will identify and quantify clinically available factors at the time of ED presentation (eg, age, mechanism of injury, symptom presence and severity, history and physical examination) associated with PCS in children following concussion.

\section{Objectives}

The goal of this study is to derive a clinical prediction rule from key factors that is predictive of the development of PCS. We will identify and quantify clinically available factors at the time of ED presentation (eg, age, mechanism of injury, symptom presence and severity, history and physical examination) associated with eventual development of PCS in children following concussion. Specific objectives will be to (1) apply standardised clinical assessments for children and adolescents following concussion across Canada; (2) determine the prevalence of PCS at 1 month follow-up in children aged 5-17 years presenting for ED care following concussion. The literature regarding concussion incidence and PCS development is speculative and not based on a standardised clinical assessment. This would be the largest epidemiological cohort of concussions in the literature to date; (3) determine the inter-rater agreement for predictors; (4) determine the association between the clinical assessment findings and PCS presence; (5) derive a sensitive clinical prediction rule for children and adolescents with concussion to predict PCS using multivariate techniques; (6) determine ED physician accuracy in predicting PCS duration without using a rule; (7) determine the association, if there is one, between self-reported cognitive symptom persistence and objective neuropsychological testing; (8) determine predictors of persistent neuropsychological symptoms; (9) determine the inter-rater agreement for these predictors; and (10) report the epidemiology of neuropsychological symptoms at 4 and 12 weeks postconcussion.

\section{METHODS}

\section{Study population}

Inclusion criteria

Participants presenting to one of the study hospital EDs after sustaining a head injury will be eligible if they

1. Are aged $5-17$ years;

2. Have a concussion, defined by Zurich consensus statement ${ }^{3}$ :

A direct blow to the head, face, neck or elsewhere on the body with an impulsive force transmitted to the head, resulting in one or more of the symptoms in one or more of the following clinical domains (which may or may not have involved loss of consciousness):

- Somatic symptoms (eg, headache, nausea, loss of balance, dizziness, sensation to light or noise, visual problems and clumsiness)

- Cognitive symptoms (eg, feeling like in a fog, difficulty concentrating or remembering, 
answering questions more slowly and confused with directions/task)

- Emotional/behavioural symptoms (eg, irritable, sad, nervous and emotional lability), physical signs (eg, loss of consciousness and amnesia)

- Sleep disturbance (eg, sleeping more, fatigue, drowsiness and insomnia);

3. Suffered the initial injury in the previous $48 \mathrm{~h}$;

4. Are proficient in English or French.

\section{Exclusion criteria}

Patients will be excluded if they present with traumatic head injuries with any of the following: (1) Glasgow Coma Scale $\leq 13$; (2) any abnormality on standard neuroimaging studies, ${ }^{3}$ including any positive head CT findings (note: neuroimaging is not required, but may be performed if believed to be clinically indicated); (3) neurosurgical operative intervention, intubation or intensive care required; (4) multisystem injuries with treatment requiring admission to hospital, operating room or procedural sedation in ED (note: admission to hospital for observation or management of ongoing concussion symptoms is not an exclusion criteria); (5) severe chronic neurological developmental delay resulting in communication difficulties; (6) intoxication at the time of ED presentation as per clinician judgment; (7) no clear history of trauma as primary event (eg, seizure, syncope or migraine as primary event); or (8) previously enrolled in this same study.

\section{Patient selection}

A consecutive sample of patients with possible concussion arriving between the hours of 12:00 and 22:00 will be screened for the study. Census data from the sites reveal that less than one-fifth of paediatric concussion patients present outside of these study hours and, therefore, do not justify the additional expense of research personnel coverage. For comparative purposes, demographic and outcome data will be collected from the ED record of treatment for eligible patients who are not enrolled into the study.

\section{Study setting}

The patients will be enrolled from the EDs of nine Canadian paediatric hospitals: IWK Health Sciences Centre (Nova Scotia), CHU Sainte-Justine (Quebec), Montreal Children's Hospital (Quebec), Children's Hospital of Eastern Ontario (Ontario), The Hospital for Sick Children (Ontario), Children's Hospital of Western Ontario (Ontario), Children's Hospital of Winnipeg (Manitoba), Stollery Children's Hospital (Alberta) and Alberta Children's Hospital (Alberta). These centres constitute 9 of the 12 paediatric hospitals in Canada and have a combined annual ED census of approximately 500000 patient-visits. All sites are active members of Pediatric Emergency Research Canada (PERC) and have successfully collaborated in many multicentre prospective studies. Formed in 1995, PERC is an established network of healthcare researchers dedicated in improving care in paediatric emergency medicine through multicentre research. The PERC network received a 2011 CIHR-CMAJ Top Achievements in Health Research Award ${ }^{32}$ and has significant experience in decision rule derivation and validation studies including the validation of decision rules for the use of radiography for children with ankle and knee injuries, and recently in the derivation and validation of a clinical decision rule regarding the need for a CT scan in children with minor head injuries. $^{33}$

\section{Standardised patient assessment \\ Patient recruitment}

When a child aged between 5 and 17 years presents to triage/registration with a head injury, the patient's chart and/or electronic patient tracking system will be flagged as a potentially study-eligible patient. Research assistants (RAs) located in the department will work collaboratively with the ED clinical team to also identify potential study participants. Prior to assessment by the physician, RAs will screen these patients for eligibility using brief screening questionnaire. If eligible, RAs will discuss the study with the patient and families. Eligible and willing parents, along with children and adolescents, capable of consenting on their own behalf will be asked for written informed consent, and those children aged 7 years or older who are unable to consent on their own behalf will be asked for assent. RAs will maintain a screening $\log$ of all potentially eligible patients during the study enrolment hours complete with reasons for exclusion. A second $\log$ will also be maintained of those potentially eligible patients who present outside of the study enrolment hours to confirm that patient characteristics are similar. These recruitment and tracking strategies have been pilot tested in our previous work and were found to be highly successful.

\section{Standardised patient assessment of concussion using a validated tool}

With the guidance of an RA, parent/guardian/child will answer a series of questions in electronic survey format in their first language (English or French) using a portable computer tablet. In addition to questions regarding patient demographics, both parents and children will also complete the validated Acute Concussion Evaluation (ACE), ${ }^{34}$ which is embedded into the Child Sports Concussion Assessment Tool 3. ${ }^{3}$ The ACE is a 22-item dichotomous evidence-based inventory for the initial evaluation and diagnosis of concussion and has been validated as an initial concussion assessment tool for parental informants of children aged 3-18 years. Data are collected on injury characteristics, symptoms checklist (physical, emotional, cognitive and sleep) and history (prior concussion, headache, developmental or psychiatric). This is to ensure that the child does have a concussion, rather than rely solely on physician judgement. By using a validated standardised assessment, we 
will ensure uniform application of the definition of concussion.

\section{Physician management and prognostication}

The treating physician will assess the child as per the normal operating procedures of ED. Once a patient has given consent to participate in the study, the RA will liaise with the treating physician to notify them that the patient is enrolled in the concussion study. The child will remain in the normal treatment queue, and participation in this study is not anticipated to lengthen their ED visit. The physician will complete a brief electronic survey on the same tablet computer. This survey will collect data about medical history, comorbidities, therapies received during the ED visit, discharge instructions and prognostication regarding symptom duration.

\section{Quality assurance}

Since data will be collected electronically via portable tablet, embedded logic safeguards will ensure that variables are entered within predetermined ranges. Warning messages will be programmed to alert for incomplete data fields. There will also be an ongoing evaluation of the quality of the patient assessments judged by compliance in enrolling eligible patients. During our pilot study, $80 \%$ of eligible patients were enrolled and, to ensure similar numbers recruited, research coordinators at each site will provide clinicians with monthly feedback regarding recruitment data as well as specific review of any individual problems that may arise. Clinicians will not, however, be given any indication of the preliminary accuracy or reliability of individual variables.

\section{Variables from history and physical examination}

The 46 potential variables selected for assessment in the study were chosen based on expert discussions during the planning meeting, our recent systematic review, previous clinical studies by our team and clinical experience. Variables selected are listed in box 1 .

These variables were felt to be most useful in predicting whether or not patients with concussion may be at risk for developing PCS. Further, to ensure that our results will be effectively compared across studies and aggregated into future meta-analyses, we will incorporate the standardised dataset recommendations from the internationally recognised National Institute of Neurological Disorders and Stroke (NINDS) Common Data Element Project. ${ }^{35}$ This National Institute for Health (NIH)-funded project established standards for clinical research within the neurological community on traumatic brain injury. This dataset utilises expert consensus approved data dictionary instructions, including the preferred format for recording.

\section{Inter-rater reliability}

All ED physicians and research staff at each participating site will be trained on data collection methods using a standardised lecture/video and training session. A study

\section{Box 1 Potential predictor variables}

Age (years)

Gender

Concussion caused by less forceful mechanism (yes/no)

History of headaches requiring treatment (yes/no)

Family history of migraine (yes/no)

History of developmental or learning problems (not severe delays; yes/no)

Previous diagnosis of attention deficit hyperactivity disorder

(yes/no)

Previous diagnosis of anxiety (yes/no)

Previous diagnosis of depression (yes/no)

History of medication for behavior or mood (yes/no)

Glasgow Coma Scale in emergency department (ED; 14 vs 15)

Pharmacotherapy in ED for pain (yes/no)

Pharmacotherapy in ED for nausea (yes/no)

Mechanism of injury (sport, motor vehicle, fall, assault or other)

Protective head gear (yes/no)

Location of impact (frontal, temporal $(\mathrm{L} / \mathrm{R})$, parietal $(\mathrm{L} / \mathrm{R})$,

occipital, neck or indirect)

Loss of consciousness

Amnesia

Seizure

Appeared dazed or stunned

Initially confused about events

Answers questions slowly

Repeats questions

Forgetful

Physical

Headache

Nausea

Vomiting

Balance problems

Dizziness

Visual problems

Fatigue

Sensitivity to light

Sensitivity to noise

Numbness/tingling

Cognitive

Mentally foggy

Feeling slowed down

Difficulty concentrating

Difficulty remembering

Emotional

Irritability

Sadness

More emotional

Nervousness

Sleep

Drowsiness

Sleeping less than normal

Sleeping more than normal

Trouble falling asleep

manual including a data dictionary will be available at each site. To measure inter-rater reliability, $5 \%$ of patients will be assessed for clinical variables by a second physician who will be blinded to the results of the first assessment. These second assessments will be performed 
in all centres on a feasibility basis whenever two physicians are available in ED.

\section{Follow-up}

Families will be asked to provide contact information prior to discharge. Depending on their preferences, enrolled patients will either be entered into the automated follow-up web survey using REDCap ${ }^{36}{ }^{37}$ or the telephone follow-up survey. For those who would prefer electronic follow-up, a link to a secure web-based questionnaire will be sent to the parental email address on day 7 following the initial injury and then 2, 4, 8 and 12 weeks postenrolment. The same schedule and questionnaires will be used for the families that opt for telephone follow-up. In the event that patients do not complete the electronic survey within $24 \mathrm{~h}$ of receipt, a second email will be sent. If there is still no response to the electronic survey, the family will be contacted by telephone for a phone interview. The follow-up questionnaires employ the validated Post-Concussion Symptom Inventory (PCSI). In addition, the follow-up survey will query symptom management, follow-up with health providers, school absenteeism, changes in academic performance, date of return to sport and school and patient/ family quality of life. Our pilot established success with web-based follow-up with $91 \%$ (89/98) of patients having requested web follow-up; only two families changed from web to telephone follow-up. Patient-level assessment of longer term outcomes (beyond 3 months) is felt to be beyond the scope of this study.

\section{Neuropsychological follow-up (optional)}

All children from four centres (CHU Sainte-Justine, Montreal Children's Hospital, Children's Hospital of Eastern Ontario, the Hospital for Sick Children) will be offered enrolment in an optional neuropsychological arm of the study at the time of their initial consent by the onsite trained RA. This will involve two return visits to the centre, at 4 and 12 weeks postconcussion. Each visit will be approximately $2.5 \mathrm{~h}$ in length, during which we will measure the mental and social capacities of children and adolescents who have suffered a concussion. These data will be gathered using questionnaires and tests in their first language (English or French) designed to measure mental skills and behaviour in two ways: (1) indicators of behaviour and functional abilities and (2) measurements of mental and social skills. These measures will be administered by an RA who is fully trained in psychometry. The results of these assessments will be reviewed and interpreted by a licensed neuropsychologist, who will follow-up with any significant clinical concerns and refer appropriately. In cases of significant clinical concerns arising at the 4-week assessment, the patient and parents will be notified. A summary written report will be issued after the completion of the 12-week assessment, again, with appropriate follow-up indicated if necessary.

\section{Primary outcome measure}

The primary outcome is the proportion of children aged 5-17 years who have PCS at 1-month follow-up. A PCS case is defined as an increase from preconcussion baseline of three or more symptoms on the validated PCSI at 1 month (consistent with the International Classification of Diseases, 10th Revision (ICD-10) definition of PCS).

\section{Post-Concussion Symptom Inventory}

PCSI is a set of symptom scales for parents (26-item, 7-point Likert scale) and developmentally specific selfreport forms for children aged 5-7 years (13 items, 3-point Likert scale), aged 8-12 years (25-item, 3-point Likert scale) and 13-year-olds to 18-year-olds (26-item, 7-point Likert scale). PCSI is the only one of the two measures applicable to younger children who have both validity and reliability data published in the literature. $^{34} 3839$ The PCSI queries symptoms reflecting physical, cognitive, emotional and sleep domains. Even with younger children, there is excellent internal consistency with PCSI (aged 5-7 years, $\mathrm{r}=0.76$; aged 8-12 years, $\mathrm{r}=0.87) .{ }^{38}$ Inter-rater agreement has also been examined comparing symptoms ratings from the child and parent. At enrolment, patients and parents will complete an inventory of symptoms that were present prior to the injury (72 $\mathrm{h}$ prior to enrolment) to establish a patient baseline. Since each case of PCS is defined as a change from an individual's baseline in three or more symptoms, total summation of subunits is not required for PCSI scoring. Therefore, it is not problematic that there are different item values or totals across age groups. Symptom severity (mild -moderate -severe) at the time of ED presentation will also be analysed as a potential predictor of symptom duration.

\section{Secondary outcomes measures}

Paediatric quality-of-life inventory (PedsQL)

Standard paediatric practice requires the involvement of parents. The role of the family is crucial to the recovery process, and delayed recovery from concussion has been shown to impact the quality of life of the patient and the family. ${ }^{9} 1617$ The PedsQL is a reliable and valid measure of health-related quality of life in healthy children and adolescents and those with acute and/or chronic health conditions. ${ }^{35}$ Parent versions exist for children aged 2-18 years (in 4 age groups) and child versions for those aged 5 years and above. The inventory covers four domains: physical, emotional, social and school $(8,5,5$ and 5 items, respectively) and takes approximately $4 \mathrm{~min}$ to complete. This secondary outcome measure will be used to determine the impact of PCS on the quality of life of patients and families.

\section{Neuropsychological evaluation}

A separate battery of neuropsychological assessment measures will be administered to those children who choose to participate in this arm of the study (see box 1). This 
battery includes measures of intelligence, language, visual-spatial/motor functions, attention, memory/ working memory, executive functioning, academic achievement as well as behavioural/socioemotional functioning.

\section{Wechsler Abbreviated Scale of Intelligence}

The Wechsler Abbreviated Scale of Intelligence is a reliable and valid measure of intellectual functioning in children and adults. ${ }^{40}$ The Wechsler Abbreviated Scale of Intelligence consists of four subtests, which provide estimates of the Full Scale IQ the Verbal IQ (based on Vocabulary and Similarities subtests) and Performance IQ (based on Block Design and Matrix Reasoning subtests). It takes approximately $30 \mathrm{~min}$ to administer this measure.

\section{Delis-Kaplan Executive Functioning System}

The Delis-Kaplan Executive Functioning System is a reliable and valid measure of executive functioning in children and adults. It consists of many subtests, of which we will use three. ${ }^{41}$ The Trails subtest measures the ability to complete several visual spatial sequences, including numbers and letters in a mixed array as quickly as possible. The Verbal Fluency subtest asks the participant to generate as many words as possible in $1 \mathrm{~min}$ that either begin with a specific letter of the alphabet, fit in a specific category, or that switches between two categories. The Color-Word Interference Test measures the participant's ability to inhibit a dominant and automatic verbal response when reading the names of colours that are printed in a variety of ink colours. It takes approximately 20 min to administer these subtests.

\section{Digit Span and Coding subtest of the Wechsler Intelligence Scale for children (fourth edition)/Wechsler Adult Intelligence Scale (fourth edition)}

The Digit Span and Coding subtests are reliable and valid measures of short-term memory/working memory and speed of information processing in children and adults. ${ }^{42-44}$ The Digit Span subtest measures the participant's ability to repeat numbers read aloud by the examiner in a forward and backward order (for participants aged 16 years or older, the ability to sequence a series of numbers will also be assessed). The Coding subtest requires the participant to reproduce symbols in a sequence as quickly as possible. It takes approximately 10 min to administer these subtests.

\section{Rey-Osterrieth Complex Figure Test}

The Rey-Osterrieth Complex Figure Test is a reliable and valid measure of visual spatial and executive functioning in children and adults. ${ }^{45}$ The participant is asked to reproduce the complex figure with it initially in front of her/him. The participant is then asked to reproduce the figure from memory both immediately after and 30 min after the initial reproduction. It takes approximately $10 \mathrm{~min}$ to administer this test.
Dynamometer (Grip Strength Test)

The dynamometer test is a reliable and valid measure of the grip strength in children and adults. ${ }^{46}$ The participant is asked to squeeze the dynamometer with the dominant hand, followed by the non-dominant hand. It takes approximately $5 \mathrm{~min}$ to administer this test.

\section{Grooved Pegboard Test}

The Grooved Pegboard Test is a reliable and valid measure of speeded eye-hand dexterity in children and adults. ${ }^{47}$ The participant is asked to place key-shaped pegs in a pegboard as quickly as possible with the dominant hand, followed by the non-dominant hand. It takes approximately $10 \mathrm{~min}$ to administer this test.

\section{Conner's Continuous Performance Test-second edition, version 5}

The Conner's Continuous Performance Test is a task-oriented computerised assessment of attentional functioning in children and adults. ${ }^{48}$ The participant is asked to respond to some characters on the computer screen and not to respond to other characters. It takes approximately $15 \mathrm{~min}$ to administer this test.

\section{California Verbal Learning Test for Children/California Verbal Learning Test-second edition}

The California Verbal Learning Test is a reliable and valid measure of verbal learning and memory in children and adults. ${ }^{49}$ A lengthy list of words is read aloud to the participant for a total of five times, with a recollection after each presentation. Long-term recollection is also assessed. It takes approximately $20 \mathrm{~min}$ to administer this test.

\section{Wechsler Individual Achievement Test—-second edition}

The Wechsler Individual Achievement Test is a reliable and valid measure of academic achievement in children and adults, normed in both official languages of Canada. ${ }^{44}$ The participant is asked to read a series of words, to respond to questions assessing reading comprehension and to complete a series of mechanical arithmetic. It takes approximately $30 \mathrm{~min}$ to administer this test.

\section{Child Behavior Checklist}

The Child Behavior Checklist is a reliable and valid parent questionnaire assessing their child's functioning in emotional, social, cognitive and behavioural domains. ${ }^{51}$ It takes approximately $20 \mathrm{~min}$ to complete this test.

\section{Conner's Rating Scale-third edition}

The Conner's Rating Scale is a reliable and valid parent questionnaire assessing their child's functioning in emotional, social, cognitive and behavioural domains. ${ }^{52}$ The emphasis of this measure is attentional in nature. It takes approximately $15 \mathrm{~min}$ to complete this test. 
Behavioral Rating Inventory of Executive Function

The Behavioral Rating Inventory of Executive Function is a reliable and valid parent questionnaire assessing their child's executive functioning. ${ }^{53}$ It takes approximately 15 min to complete this test.

\section{Strength and Difficulties Questionnaire}

The Strength and Difficulties Questionnaire is a reliable and valid parent questionnaire assessing their child's emotional symptoms, conduct problems, hyperactivity/ inattention, peer relationships and prosocial behaviour. ${ }^{54}$ It takes approximately $10 \mathrm{~min}$ to complete this test.

\section{Data analysis}

Inter-rater agreement

The inter-rater agreement for each variable will be assessed using a $\kappa$ coefficient, the proportion of potential agreement beyond chance, along with $95 \%$ CIs. ${ }^{55}{ }^{56}$ For variables with three or more ordered categories, a weighted $\mathrm{\kappa}$ measure of inter-rater agreement will be calculated ${ }^{57} \mathrm{~A}$ variable will be deemed to have an acceptable agreement if the $\kappa$ coefficient has a value of at least $0.6 .^{55} 56$

\section{Univariate analysis}

Univariate analyses will be used to determine the strength of association between each variable and the primary outcome, PCS. This process will aid in the selection of the best variables for the multivariate analyses. The appropriate univariate technique will be chosen according to the type of data: for nominal data, a $\chi^{2}$ test with continuity correction; for ordinal variables, a Mann-Whitney U test; and, for continuous variables, an unpaired two-tailed t test, using pooled or separate variance estimates, as appropriate.

\section{Multivariate analysis}

Multivariate analysis will be used to derive a predictive model for PCS. Variables found to be both reliable $(\kappa>0.6)$ and independently associated with the outcome $(\mathrm{p}<0.2)$ will be used as input to a multivariate logistic regression. Model fit will be assessed using the Hosmer-Lemeshow test and Nagelkerke's pseudo $-\mathrm{R}^{2}$.

An alternate strategy for deriving a predictive model is recursive partitioning. This approach has been recommended for deriving highly sensitive predictive models, while logistic regression may produce better predictive accuracy ${ }^{58}$ However, high sensitivity is not the primary goal in the proposed study. Further, a fitted logistic regression model has the advantage that it can be used to derive a risk score using a points system. ${ }^{59}$ For these reasons, logistic regression was chosen as the primary analytic approach.

The objective of the logistic regression analysis will be to find the best combinations of variables for accurately predicting PCS. Receiver operating characteristic (ROC) curves will be used to assess the trade-off between sensitivity and specificity based on applying different probability thresholds to the fitted model. The derived models must be easy to use by clinicians and therefore should contain as few variables as possible. As described in a recent systematic review of paediatric clinical prediction rules, 'Seeking $95 \%$ sensitivity for clinical prediction rules for child health conditions may be an elusive and counterproductive goal, especially when the sensitivity of a less-than-perfect clinical prediction rule is superior to a clinician's judgement alone (Ref. 60, p.e671)'. Assuming more than one model meets the minimum acceptable criteria, the best model will be the one which has the highest positive predictive value/sensitivity and the fewest number of component variables.

\section{Classification performance}

The derived prediction rule will be cross validated by comparing the classification of each patient to their actual status for the primary outcome. This will allow an estimate, with 95\% CIs computed using the Wilson score method, of the sensitivity and specificity of the rule. A more robust validation will be carried out prospectively on a new set of patients in phase II.

\section{Physician judgement}

Physicians will be asked to predict likelihood of PCS using a Likert scale. Data from those predictions will be tabulated in descriptive format. Information on the predicted probabilities will be used to calculate ROC curves and likelihood ratios for determining the duration of symptom persistence. The accuracy of the physicians' predictions will be compared to that of the derived prediction rule by the ROC curve analysis.

\section{Sample size}

A well-supported recommendation for the development of a predictive model is that 10 events are required for each candidate variable. ${ }^{6162}$ Further, a recent systematic review examining the methodology of clinical prediction rule studies endorses this recommendation. ${ }^{63}$ On the basis of preliminary work, we identified 46 potential predictor variables (see box 1). Our pilot demonstrated that $25 \%$ of patients had PCS (as per ICD-10 definition of 3 or more symptoms at 1 month). After screening for interrater agreement (we assumed a $25 \%$ dropout of variables based on our CT Head Rule derivation ${ }^{33}$ ), we would require 345 PCS cases (10 cases per predictor variable after $\kappa$ screening). In order to obtain 345 PCS cases, we would need to enrol 1380 new concussions. Assuming a comparable $23 \%$ loss to follow-up at 1 month, we would require a sample size of 1792 patients.

\section{Methodological considerations}

\section{Alternate definition of PCS case considered}

The timing discrepancy between Diagnostic and Statistical Manual of Mental Disorders, Fourth Edition (DSM-IV) and ICD-10 definitions of PCS is conceded to be a shortcoming in the literature. ${ }^{12}$ While DSM-IV criteria define a case as persistent symptoms at 3-month duration, experts at our 2-day planning meeting 
determined that 1 month of persistent symptomatology was the most clinically relevant outcome (ICS-10 criteria). Both definitions require the presence of multiple symptoms as compared to baseline. Preliminary data from our pilot study confirmed that most children with PCS have changes from baseline for multiple symptoms rather than for just one or two symptoms.

\section{Alternate analysis considered}

Recursive partitioning has been widely used in the development of clinical prediction rules and recursive partitioning may be more suitable than logistic regression when the objective is to correctly classify one outcome group at the expense of the other. ${ }^{33}$ Recursive partitioning creates a binary decision tree by automatically splitting on the values of input variables so as to achieve optimal classification of an outcome variable. ${ }^{64}$ However, predictive models are likely better developed as risk scales with points based on logistic regression analyses. The use of risk scales gives the probability of outcomes; clinicians are then able to choose their own cut points for action.

\section{ETHICS AND DISSEMINATION}

This study poses little to no risk to the participating patients and their families. Patients will receive standard care in ED. Participation in the study will not negatively impact or restrict them from receiving additional assessments, investigations (CT or MRI), consultations or management, as determined by treating physicians. Risks to the patient and the family only exist in the realm of security and privacy of their data including responses to various questionnaires used to identify prognosticators for PCS. Research personnel will take all appropriate and customary steps to ensure that data remain secure and that patient privacy and confidentiality are maintained. All patients and families will provide written informed consent/assent and will have the ability to withdraw at any time without explanation.

Results will be disseminated at international conferences and in manuscripts to peer-reviewed journals. Publications topics will include epidemiology of PCS at 1-month follow-up in children aged 5-17 years presenting for ED care following concussion (the largest epidemiological cohort of concussions in the literature to date; derivation of a sensitive clinical prediction rule for children and adolescents with concussion to predict PCS using multivariate techniques; epidemiology of neuropsychological symptoms at 1 and 3 months postconcussion; and the association between self-reported cognitive symptom persistence and objective neuropsychological testing.

We need rigorous evidence about incidence of PCS in children and its impact on the quality of life for both children and families. Validated, easy-to-use prognosticators do not exist for clinicians to identify children with concussion who are at the highest risk for PCS and sequelae. Physicians currently lack the ability to accurately inform children and parents whether they should expect longer symptoms. A clinical prediction rule would allow physicians to determine who would most likely benefit from potential interventions, allow physicians to quickly initiate best care post-mild traumatic brain injury and permit concussion research to move forward.

With the identification of high-risk patients for PCS, clinical trials may start to ameliorate or possibly prevent persistent symptomatology. The literature is ripe with promising pilot trials of pharmacotherapeutic interventions in patients who have suffered moderate-to-severe traumatic brain injury (eg, amantadine, progesterone or magnesium $)^{65-67}$ as well as promising rehabilitation interventions. ${ }^{68}{ }^{69}$ Prior to a large, randomised, double-blind interventional trial in children and adolescents presenting with a concussion, it is imperative for ethical considerations that only high-risk patients be selected for trials of pharmacotherapeutic agents with potential for significant side-effect profiles. ${ }^{70}$ Further, other than expert consensus on graduated return-to-play guidelines, there is a minimal trial-based evidence regarding the ideal non-pharmacological or rehabilitation management of persistent symptoms; our study is fundamental to future interventional trials. We still do not know the ideal approaches to returning to school and sport. ${ }^{1}{ }^{70}$ Results from our study will permit future concussion trials to answer these and similar questions by targeting patients most likely to benefit. In short, our proposed research is necessary to allow concussion research to move forward.

Further, evidence supports the benefit of early education with coping mechanisms to reduce PCS. ${ }^{71}{ }^{72}$ Therefore, front-line primary care and emergency physicians need to be able to provide accurate counselling to families and patients following concussion and timely specialist referral based on risk stratification. Our pilot data demonstrated that parental anxiety remained amplified, while their children remained symptomatic. ${ }^{73}$ Results from this proposed study could be used to improve anticipatory guidance to parents and to offer expectation management and coping mechanisms.

Finally, despite improved recognition of sport-related concussion in adolescents over the past decade, little attention has been given to younger children. ${ }^{38}$ Our study will generate epidemiological data on younger children and other overlooked populations (eg, non-sports-related concussions, girls, minor behavioural or developmental problems, such as Attention-deficit hyperactivity disorder).

Collaborators Pediatric Emergency Research Canada (PERC) Concussion Team: Peter Anderson, PhD, Children's Hospital of Eastern Ontario, Psychology, Karen Barlow, MD, Alberta Children's Hospital, Pediatric Neurology, Miriam Beauchamp, PhD University of Montreal, Psychology, Darcy Beer, MD, Manitoba Children's, Pediatric Emergency, Kathy Boutis, MD, Hospital for Sick Children, Pediatric Emergency, Brian J Brooks, PhD, Alberta Children's Hospital, Clinical Neurosciences Psychology, Emma Burns, MD, IWK, Pediatric Emergency, William Craig, MD, Stollery, Pediatric Emergency, Carol DeMatteo, MSc, McMaster University, School of Rehabilitation Science, Sasha Dubrovsky, MD, Montreal Children's Hospital, Pediatric Emergency, Ken Farion, MD Children's Hospital of Eastern Ontario, Pediatric Emergency, 
Stephen Freedman, MD, Alberta Children's Hospital, Pediatric Emergency, Isabelle Gagnon, PhD, Montreal Children's Hospital, Department of Pediatrics, Jocelyn Gravel, MD, Hospital Ste. Justine, Pediatric Emergency, Blaine Hoshisaki, PhD, University of Ottawa, Kinesiology, Michelle Keightley, PhD, University of Toronto, Psychology, Terry Klassen, MD, Manitoba, Pediatric Emergency, William Meehan, MD, Harvard University, Sports medicine, Willem Meeuwisse, MD, University of Calgary, Sports medicine, Angelo Mikrogianakis, MD, Alberta Children's Hospital, Pediatric Emergency, Lawrence Richer, MD, Stollery, Pediatric Neurology, Gurinder Sangha, MD, London, Pediatric Emergency, Michael Vassilyadi, MD, Children's Hospital of Eastern Ontario, Pediatric Neurosurgery.

Contributors RZ, MO and NB led the study concept and design, selected outcome measures and involved in writing of the manuscript. RZ wrote the first draft of the manuscript. All authors read, critically revised and approved the final version of the manuscript.

Funding This study is supported by a Canadian Institute of Health Research (CIHR) Operating Grant (MOP: \#126197) as well as by a Canadian Institute of Health Research (CIHR) mild Traumatic Brain Injury Team Grant (TM1: \#127047).

\section{Competing interests None.}

Ethics approval Ethics has been obtained through the Children's Hospital of Eastern Ontario Research Ethics Board (No 13/94X).

Provenance and peer review Not commissioned; internally peer reviewed.

Open Access This is an Open Access article distributed in accordance with the Creative Commons Attribution Non Commercial (CC BY-NC 3.0) license, which permits others to distribute, remix, adapt, build upon this work noncommercially, and license their derivative works on different terms, provided the original work is properly cited and the use is non-commercial. See: http:// creativecommons.org/licenses/by-nc/3.0/

\section{REFERENCES}

1. Rivara F. Concussion: time to start paying attention. Arch Pediatr Adolesc Med 2012;166:666-7.

2. Meehan W, Bachur R. Sport-related concussion. Pediatrics 2009;123:114-23.

3. McCrory P, Meeuwisse W, Aubry M, et al. Consensus statement on concussion in sport: the 4th International Conference on Concussion in Sport held in Zurich, November 2012. Br J Sports Med 2013;47:250-8.

4. Meehan W, Mannix R. Pediatric concussions in United States emergency departments in the years 2002 to 2006. J Pediatr 2010;157:889-93.

5. Emery $\mathrm{C}$, Kang J, Shrier I, et al. Risk of injury associated with bodychecking experience among youth hockey players. CMAJ 2011;183:1249-56.

6. National Center for Injury Prevention and Control. Traumatic brain injury in the United States: emergency department visits, hospitalizations and deaths 2002-2006. Atlanta, GA: Centers for Disease Control and Prevention, 2010

7. Langlois J, Rutland-Brown W, Wald M. The epidemiology and impact of traumatic brain injury. J Head Trauma Rehabil 2006;21:375-8.

8. National Center for Injury Prevention and Control. Report to Congress on mild traumatic brain injury in the United States: steps to prevent a serious public health problem. Atlanta, GA: Centers for Disease Control and Prevention, 2003.

9. Yeates KO, Kaizar E, Rusin J, et al. Reliable change in postconcussive symptoms and its functional consequences among children with mild traumatic brain injury. Arch Pediatr Adolesc Med 2012;166:615-22.

10. Belanger $\mathrm{H}$, Vanderploeg $\mathrm{R}$. The neuropsychological impact of sports-related concussion: a meta-analysis. J Int Neuropsychol Soc 2005;11:345-57.

11. Zafonte R. Diagnosis and management of sports-related concussion. JAMA 2011;306:79-86.

12. Ayr L, Yeates $\mathrm{K}$, Taylor $\mathrm{H}$, et al. Dimensions of postconcussive symptoms in children with mild traumatic brain injuries. J Int Neuropsychol Soc 2009;15:19-30.

13. Burton L, Quinn B, Pratt-Cheney J, et al. Headache etiology in a pediatric emergency department. Pediatr Emerg Care 1997;13:1-4.
14. Yeates K, Luria J, Bartkowski H, et al. Postconcussive symptoms in children with mild closed head injuries. J Head Trauma Rehabil 1999;14:337-50.

15. Barlow K, Crawford S, Stevenson A, et al. Epidemiology of postconcussion syndrome in pediatric mild traumatic brain injury. Pediatrics 2010;126:e374-81.

16. Carroll L, Rosner D. The concussion crisis: anatomy of a silent epidemic. Simon \& Schuster, 2011.

17. Scorza K, Raleigh M, O'Connor F. Current concepts in concussion: evaluation and management. Am Fam Physician 2012;85:123-32.

18. Korinthenberg R, Schreck J, Weser J, et al. Post-traumatic syndrome after minor head injury cannot be predicted by neurological investigations. Brain Dev 2004;26:113-17.

19. Babikian T, Satz P, Zaucha K, et al. The UCLA Longitudinal Study of neurocognitive outcomes following mild pediatric traumatic brain injury. J Int Neuropsychol Soc 2011;17:886-95.

20. Forsyth R, Kirkham F. Predicting outcome after childhood brain injury. CMAJ 2012;184:1257-64.

21. Anderson V, Brown S, Newitt $\mathrm{H}$, et al. Educational, vocational, psychosocial, and quality-of-life outcomes for adult survivors of childhood traumatic brain injury. J Head Trauma Rehabil 2009;24:303-12.

22. Anderson $\mathrm{V}$, Jacobs $\mathrm{R}$, Spencer-Smith $\mathrm{M}$, et al. Does early age at brain insult predict worse outcome? Neuropsychological implications. J Pediatr Psychol 2010;35:716-27.

23. Meehan W, Zhang J, Mannix R, et al. Increasing recovery time between injuries improves cognitive outcome after repetitive mild concussive brain injuries in mice. Neurosurgery 2012;71:885-91.

24. Guskiewicz K, Marshall S, Bailes J, et al. Association between recurrent concussion and late-life cognitive impairment in retired professional football players. Neurosurgery 2005;57:719-26.

25. Gaetz M, Goodman D, Weinberg H. Electrophysiological evidence for the cumulative effects of concussion. Brain Inj 2000;14:1077-88.

26. Gronwall D, Wrightson P. Cumulative effects of concussion. Lancet 1975;2:995-7.

27. Matser E, Kessels A, Lezak M, et al. Cumulative problems with memory and planning in amateur soccer. JAMA 1999;282:971-3.

28. Marchie A, Cusimano M. Bodychecking and concussions in ice hockey: should our youth pay the price? CMAJ 2003;169:124-8.

29. Swaine B, Tremblay C, Platt R, et al. Previous head injury is risk factor for subsequent head injury in children: a longitudinal cohort study. Pediatrics 2007;119:749-58.

30. Zemek R, Farion K, Sampson M, et al. Prognosticators of persistent symptoms following pediatric concussion: a systematic review. JAMA Pediatr 2013;167:259-65.

31. Callahan J. Pediatric concussions in United States emergency departments: the tip of the iceberg. J Pediatr 2010;157:873-5.

32. Kelsall D. Celebrating top achievements in health research 2011 CMAJ 2012;184:189-91.

33. Osmond M, Klassen T, Wells G, et al. CATCH: a clinical decision rule for the use of computed tomography in children with minor head injury. CMAJ 2010;182:341-8.

34. Gioia G, Schneider J, Vaughan C, et al. Which symptom assessments and approaches are uniquely appropriate for paediatric concussion? Br J Sports Med 2009;43(Suppl 1):i13-22.

35. Adelson PD, Pineda J, Bell MJ, et al. Common data elements for pediatric traumatic brain injury: recommendations from the working group on demographics and clinical assessment. J Neurotrauma 2012;29:639-53.

36. Harris $\mathrm{P}$, Taylor $\mathrm{R}$, Thielke $\mathrm{R}$, et al. Research electronic data capture (REDCap): a metadata-driven methodology and workflow process for providing translational research informatics support. J Biomed Inform 2009:42:377-81.

37. Vanderbilt University. REDCap. http://project-redcap.org/ Published January 2012 (accessed Sep 2012).

38. Gioia $\mathrm{G}$, Isquith $\mathrm{P}$, Schneider J, et al. New approaches to assessment and monitoring of concussion in children. Top Lang Disord 2009;29:266-81.

39. Gioia G, Janusz J, Isquith $P$, et al. Psychometric properties of the parent and teacher Post-Concussion Symptom Inventory (PCSI) for children and adolescents. J Int Neuropsychol Soc 2008;14(Suppl 1):204.

40. Wechsler D. Wechsler abbreviated scale of intelligence-Second Edition Manual. Bloomington, MN: Pearson, 2011.

41. Delis DC, Kaplan E, Kramer JH. Delis-Kaplan Executive Function System (D-KEFS). San Antonio, TX: The Psychological Corporation 2001.

42. Wechsler D. The Wechsler Intelligence Scale for Children-Fourth Edition. San Antonio: Pearson Assessment, 2004 
43. Wechsler D. The Wechsler Adult Intelligence Scale-Fourth Edition. San Antonio: Pearson Assessment, 2008.

44. Wechsler D. The Wechsler Individual Achievement Test-Second Edition. San Antonio: Pearson Assessment, 2001.

45. Osterrieth PS. Le test de copie d'une figure complex: Contribution à l'étude de la perception et de la memoir. Arch Psychol 1944;30:286-356.

46. Dynamometer-Lafayette Instruments, Lafayette, IN, USA.

47. Grooved Pegboard Test—Lafayette Instruments, Lafayette, IN, USA

48. Conners CK. Conners' Continuous Performance Test II (CPT II V.5). Toronto: MHS, 2004

49. Delis DC, Kramer JH, Kaplan E, et al. California Verbal Learning Test-Second Edition. San Antonio: Pearson, 2000.

50. Delis DC, Kramer JH, Kaplan E, et al. California Verbal Learning Test-Children's Version. San Antonio: Pearson, 1994.

51. Achenbach TM, Rescorla LA. Manual for the ASEBA School-Age Forms \& Profiles. Burlington, VT: University of Vermont, Research Center for Children, Youth, \& Families, 2001.

52. Conners CK. Conners, 3rd edition. Toronto: MHS, 2008.

53. Gioia GA, Isquith PK, Guy SC, et al. Behavioral rating inventory for executive functioning. Lutz, FL: PAR, 2000.

54. Goodman R. The extended version of the Strengths and Difficulties Questionnaire as a guide to child psychiatric caseness and consequent burden. $J$ Child Psychol Psychiatry 1999;40:791-801.

55. Kramer MS, Feinstein AR. Clinical biostatistics: LIV. The biostatistics of concordance. Clin Pharmacol Ther 1982;29:111-23.

56. Landis JR, Koch GG. The measurement of observer agreement for categorical data. Biometrics 1977;33:159-74.

57. Fleiss JL. Statistical methods for rates and proportions. 2nd edn. New York: John Wiley \& Sons, 1981.

58. Stiell I, Wells G. Methodologic standards for the development of clinical decision rules in emergency medicine. Ann Emerg Med 1999;33:437-47.

59. Sullivan L, Massaro J, D'Agostino R. Presentation of multivariate data for clinical use: the Framingham study risk score functions. Stat Med 2004;23:1631-60.
60. Maguire J, Kulik D, Laupacis A, et al. Clinical prediction rules for children: a systematic review. Pediatrics 2011;128:e666-77.

61. Harrell F, Lee K, Mark D. Multivariable prognostic models: issues in developing models, evaluating assumptions and adequacy, and measuring and reducing errors. Stat Med 1996;15(4):361-87.

62. Peduzzi P, Concato J, Kemper E, et al. A simulation study of the number of events per variable in logistic regression analysis. J Clin Epidemiol 1996;49:1373-9.

63. Bouwmeester W, Zuithoff N, Mallett S, et al. Reporting and methods in clinical prediction research: a systematic review. PLoS Med 2012;9:e1001221.

64. Breiman L, Friedman J, Olshen R, et al. Classification and regression trees.Belmont, $\mathrm{CA}$, : Wadsworth, 1983.

65. Giacino J, Whyte J, Bagiella $\mathrm{E}$, et al. Placebo-controlled trial of amantadine for severe traumatic brain injury. $N$ Engl $\mathrm{J} \mathrm{Med}$ 2012;366:819-26.

66. Junpeng M, Huang S, Qin S. Progesterone for acute traumatic brain injury. Cochrane Database Syst Rev 2011;(1):CD008409.

67. Sen A, Gulati A. Use of magnesium in traumatic brain injury. Neurotherapeutics 2010;7:91-9.

68. Gagnon I, Galli C, et al. Active rehabilitation for children who are slow to recover following sport-related concussion. Brain Inj 2009:23:956-64.

69. Leddy JJ, Kozlowski K, et al. A preliminary study of subsymptom threshold exercise training for refractory post-concussion syndrome. Clin J Sport Med 2010;20:21-7.

70. Meehan W. Medical therapies for concussion. Clin Sports Med 2011;30:115-24.

71. Ponsford J, Willmott C, Rothwell A, et al. Impact of early intervention on outcome following mild head injury in adults. J Neurol Neurosurg Psychiatry 2002;73:330-2.

72. Ponsford J, Willmott C, Rothwell A, et al. Impact of early intervention on outcome after mild traumatic brain injury in children. Pediatrics 2001:108:1297-303.

73. Zemek R, Clarkin C, Farion F, et al. Parental anxiety at initial ED presentation is not predictive of prolonged symptoms following pediatric concussion. Acad Emerg Med (accepted 6 Jun 2013). 


\section{Correction}

Zemek R, Osmond MH, Barrowman N, et al. Predicting and preventing postconcussive problems in paediatrics (5P) study: protocol for a prospective multicentre clinical prediction rule derivation study in children with concussion. BMJ Open 2013;3:e003550. One of the collaborators' initials is incorrect. Brian J Brooks should appear as Brian L Brooks.

BMJ Open 2013;3:e003550corr1. doi:10.1136/bmjopen-2013-003550corr1 